\title{
Influence of different PEEP levels on electrical impedance tomography findings in patients under general anesthesia ventilated in the lateral decubitus position
}

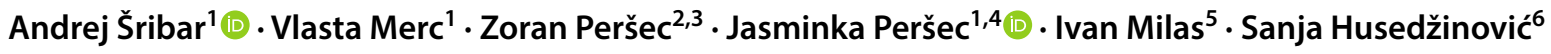

Received: 17 January 2019 / Accepted: 30 April 2019 / Published online: 6 May 2019

C) Springer Nature B.V. 2019

\begin{abstract}
To determine the effect of various PEEP levels on electrical impedance tomography (EIT) measured differences in regional ventilation, hemodynamics, lung mechanics and parameters of alveolar gas exchange. Thirty three patients scheduled for elective urologic surgery in general anesthesia in lateral decubitus position were randomized into three groups-PEEP 0,5 and 10 mbar. EIT recording, arterial blood gas analysis and hemodynamic parameters were captured at three timepointsbefore induction (T0), 5 min after lateral positioning (T1) and 90 min after positioning (T2). Dynamic compliance (Cdyn) was measured at T1 and T2. Offline EIT data analysis was performed to calculate EIT derived parameters of ventilation distribution. Patients ventilated with PEEP of $10 \mathrm{mbar}$ had a significantly lower A-a (alveolo arterial) gradient over measurements and symmetrical distribution of ventilation measured by EIT. There was no significant difference in Cdyn, center of ventilation indices and inhomogeneity index between groups. There was no difference of mean arterial pressure, cardiac index and heart rate between groups. Patients with 5 mbar of PEEP had higher stroke volume index compared to 0 and $10 \mathrm{mbar}$ at baseline and over measurements. Nondependent/dependent TV ratio as well as global inhomogeneity index were correlated with A-a gradient. Dynamic compliance showed no correlation to A-a gradient. In our study, a PEEP level of 10 mbar improved alveolar gas exchange without compromising hemodynamic stability in patients mechanically ventilated in the lateral decubitus position. EIT measured parameters may be used to determine optimal ventilation parameters in these patients with inhomogeneous lung mechanics. Further studies are needed in patients with various lung pathologies.
\end{abstract}

Keywords Mechanical ventilation · Electrical impedance tomography $\cdot$ Lateral position $\cdot$ Urology $\cdot$ PEEP

\section{Introduction}

Andrej Šribar

andrej.sribar@gmail.com

1 Clinical Department of Anesthesiology, Resuscitation and Intensive Care Medicine, University Hospital Dubrava, Av. Gojka Šuška 6, 10000 Zagreb, Croatia

2 Department of Urology, University Hospital Dubrava, Zagreb, Croatia

3 Department of Biomedical Sciences, University North, Varaždin, Croatia

4 School of Dental Medicine, Zagreb University, Zagreb, Croatia

5 Clinical Department of Urology, University Hospital Center Zagreb, Zagreb, Croatia

6 Zagreb Dental Polyclinic, Zagreb, Croatia
Patients undergoing certain urologic (such as nephrectomy or adrenalectomy) or orthopedic procedures (such as hip replacement or shoulder laparoscopy) in general anesthesia are mechanically ventilated in lateral decubitus position. After induction of general anesthesia (which initially decreases functional residual capacity), turning the patient into lateral decubitus position causes compression of dependent hemidiaphragm by abdominal organs and basal parts of the dependent lung may become atelectatic. Because all of the above, nondependent lung's compliance curve shifts to a more favorable part compared to dependent lung which may precipitate shunting $\left(\dot{V} / \dot{Q}\right.$ mismatch), drop in $\mathrm{PaO}_{2} / \mathrm{FiO}_{2}$ (Horowitz quotient) and an increase in alveolar-arterial oxygen pressure gradient (A-a gradient). These differences between the non-dependent and dependent lung are even more pronounced when the 
non-dependent pleura has been opened (for example during thoracic procedures) [1].

Other than ventilation in lateral decubitus position, factors that also cause an increase in A-a gradient are acute respiratory distress syndrome (ARDS), pulmonary embolism, right-left cardiac shunts, atelectases, obstructive lung disease, interstitial lung disease [2] and obesity [3].

Use of positive end expiratory pressure (PEEP) during mechanical ventilation is a well-established method of reducing atelectasis and shunting in dependent regions, as well as avoiding cyclical alveolar opening and closing (atelectotrauma) in adjacent regions, especially in patients with ARDS [4]. However, although PEEP may improve oxygenation, it must be used judiciously in order to avoid consequences of hyperinflated alveoli-hemodynamic compromise and dead space increase [5]. Therefore, more than 40 years ago "optimal" PEEP level was proposed as a compromise between adequate oxygenation and hemodynamic stability [6]. Many methods are in use to determine the desired PEEP levels, be it PEEP/FiO ${ }_{2}$ tables proposed by the ARDS network [7], determination of optimal compliance of the respiratory system using pressure-volume curves, estimating the transpulmonary pressure by measuring pressure in the airways and esophagus (as a surrogate of pleural pressure), lung ultrasonography [8], computerized tomography and electrical impedance tomography (EIT) [4, 5].

In recent years, development of EIT of the lung, as a noninvasive, continuous, functional imaging method has gained traction in intensive care medicine. It works on the principle of electrode belt placed around chest through which weak alternating current is applied, impedance differences between each electrode pair are calculated and then a tomogram is generated which shows spatial distribution of ventilation. In various studies, EIT has been validated as an efficient and safe method of PEEP optimization in animals and humans, especially in patients with ARDS [9-11], for laparoscopic procedures [12-14] and for one lung ventilation [15]. Certain EIT derived indices such as center of ventilation [16] and GI index [10,17] are being used in clinical practice to further assess distribution of ventilation and quantify the image.

Goal of this study is to determine effect of various levels of PEEP (0, 5, and 10 mbar) on differences in EIT measured parameters, patient hemodynamics, lung mechanics and parameters of alveolar gas exchange in patients that are ventilated in lateral decubitus position.

\section{Materials and methods}

Study was designed as a monocentric, prospective, randomized, single-blinded study. Study protocol was approved by institutional ethics board and is also registered at www. clinicaltrials.gov website with identifier NCT03704038.
Patients older than 18 years scheduled for elective urologic surgery (nephrectomy and adrenalectomy) in lateral decubitus position were included in this study.

Exclusion criteria were age less than 18 years, pregnancy, presence of cardiac electrostimulator (pacemaker), pneumonia requiring hospitalization in last 6 months, disseminated malignant disease, history of organ transplant, obstructive and/or restrictive lung disease, pulmonary hypertension and history of pulmonary embolism. Patients in which pleura was opened during surgery were excluded from the study.

Eligible patients were explained the study protocol in the anesthesia preparation room, and after signing the informed consent form were randomized into three groups (PEEP 0 mbar, PEEP 5 mbar and PEEP $10 \mathrm{mbar}$ ). After randomization patients were transferred into the operating theater where non-dominant arm radial artery was cannulated after skin infiltration with 2\% lidocaine (Flowswitch cannula, Becton Dickinson, New Jersey, USA). After arterial cannulation, EIT belt consisting of 16 electrodes (was placed at the level of 5th intercostal space and EIT measurement using Draeger Pulmovista 500 monitor (Draeger, Lubeck, Germany) was initiated. Patients were then monitored using arterial line via estimated continuous cardiac output monitor (Nihon Kohden Vismo, Nihon Kohden, Tokyo, Japan) which uses demographic data and pulse wave transit time (PWTT) to calculate cardiac output and stroke volume (and their indexed values) as well as pulse pressure variation (PPV).

Initial (baseline, T0) values were then recorded-arterial blood gas analysis which was used to calculate alveolararterial oxygen gradient in $\mathrm{kPa}$ according to the formula $A-a=\left(\mathrm{FiO}_{2} \times(p A t m-6.27)-\frac{P a_{\mathrm{CO} 2}}{0.8}\right)-\mathrm{Pa}_{\mathrm{O} 2}$ and $\mathrm{PaO}_{2} /$ $\mathrm{FiO}_{2}$ ratio in mmHg; hemodynamic parameters (heart rate, systolic and diastolic blood pressure, cardiac index, stroke volume index) and a $60 \mathrm{~s}$ EIT recording at $20 \mathrm{~Hz}$ sampling rate using low pass filter set to $<40 /$ min was performed after electrode belt calibration.

After baseline values were recorded, induction of anesthesia was initiated using fentanyl $3 \mathrm{mcg} / \mathrm{kg}$ body weight (BW), propofol $1.5 \mathrm{mg} / \mathrm{kg}$ BW and rocuronium bromide $0.6 \mathrm{mg} /$ $\mathrm{kg} \mathrm{BW}$. Patients were intubated using endotracheal tubes of inner diameter $7.5-85 \mathrm{~mm}$ according to weight and height and correct tube placement was assessed using auscultation and EIT visualization of ventilation. After intubation patients were connected to anesthesia workstation (Primus, Draeger, Lubeck, Germany) and mechanical ventilation was initiated at $8 \mathrm{ml} / \mathrm{kg} / \mathrm{PBW}$ (predicted body weight adjusted for height and gender) with PEEP predetermined by group randomization and frequency adjusted to achieve et $\mathrm{CO}_{2}$ between 4.0 and $4.5 \mathrm{kPa}$ at 1:2 I:E ratio. They were anesthetized using oxygen/air mixture of $40 \%$ oxygen and sevoflurane in concentrations adjusted to maintain minimal alveolar concentration value of 1.2. During surgery, fentanyl and rocuronium 
boluses were administered according to attending anesthesiologist's clinical assessment. Fluid administration during surgery was guided by PPV which was maintained below $15 \%$.

Five minutes after patients were placed in lateral decubitus position, all the measurements that were performed at baseline were made again (T1), as well as 90 min after positioning the patient in lateral decubitus position (T2).

At the end of surgery, patients were administered $1 \mathrm{mg}$ atropine-sulphate and $2.5 \mathrm{mg}$ neostigmine-methylsulphate for neuromuscular blockade reversal, were extubated in the operating theater and placed in post anesthesia care unit (PACU) per institutional protocol.

\subsection{Offline EIT data analysis}

One minute EIT recordings made at various timepoints (T0, T1, T2) using Pulmovista 500 monitor for each patient were imported as .eit binary files into Draeger EIT analysis tool v6.3 (Draeger, Lubeck, Germany) and then analysed and exported as .asc files which were imported as comma separated value files (.csv) into Microsoft Excel 2016 (Microsoft, Redmond, USA) where further analysis was performed.

The EIT tidal matrix consists of $32 \times 32$ pixels, and for each pixel, tidal variation (TV) is calculated as difference between end tidal and end expiratory impedance. TV regions of interest (ROI) were defined as 4 regions sized 8x32 pixels placed in a left-right axis from nondependent (ROI1 and ROI2) to dependent lung (ROI3 and ROI4) - depending on patient orientation during surgery. All regional tidal variations (i.e. for each breath) recorded during 1 min periods were averaged and nondependent/dependent TV ratio was defined as $\frac{T V_{R O I 1}+T V_{R O I 2}}{T V_{R O I 3}+T V_{R O I 4}}$. Ratio of 1 is characteristic for perfectly symmetrical ventilation, which is rarely achieved.

During each recording period, tidal images for each breath (recorded as $32 \times 32$ matrix) were averaged, and the resulting tidal image was used to calculate the vertical center of ventilation index $(\mathrm{vCoV})$ according to the formula $\mathrm{vCoV}=\frac{\Sigma\left(y_{i} \times I_{i}\right)}{\Sigma I_{i}} \times 100 \%[18,19]$ where $y_{i}$ corresponds to the pixel height of pixel $i$ scaled so the most ventral row is $0 \%$ and most dorsal row is $100 \%$. In a similar manner to $\mathrm{vCoV}$, horizontal center of ventilation (hCoV) was calculated from tidal images using $0 \%$ for most nondependent row $(0 \%)$ up to $100 \%$ for most dependent row.

Tidal image matrix was also used to calculate the GI [10], a parameter which summarizes differences in regional impedance between various lung regions. It is calculated by dividing the sum of absolute values of individual pixel TV deviations from median lung TV by sum of each pixel's TV, according to the formula GI $=\frac{\Sigma\left|I_{i}-\operatorname{Median}\left(I_{\text {lung }}\right)\right|}{\Sigma I_{i}}$ where $I_{i}$ denotes impedance levels for each individual pixel [19].

\subsection{Statistical analysis}

Sample size of 33 patients was calculated from a pilot of nine patients, with calculated effect size of 0.57 to achieve $\alpha$ error probability of 0.05 and power of 0.8 . Patients from the pilot study were not included in final data analysis.

Data is presented as tables and charts. Continuous variables are presented as mean and standard deviation. Normality of distribution was tested using Shapiro-Wilk test. Differences in categorical variables were tested using $\chi^{2}$ test. Difference between continuous variables were, dependent on normality, tested using analysis of variance (ANOVA) or Kruskal-Wallis test. Differences in repeated measurements between groups were tested using repeated measurement analysis of variance (RM-ANOVA) with between and within group interactions and post hoc Bonferroni correction. Spearman's rank correlation coefficient $(\rho)$ was used to assess the correlation between continuous variables. Sample size calculation was conducted using G*Power 3.1 [20]. Statistical analysis was performed using JASP v0.9.1 [21], jamovi v0.9.5 [22] and PAST v3.20 [23]. p values less than 0.05 were considered statistically significant.

\section{Results}

Thirty three patients were included in this study, divided into three groups (PEEP 0, 5 and 10 mbar) with 11 patients in each group. There were ten females and 23 males. Mean age was $62.1 \pm 12.9$ years, mean BMI was $27.74 \pm 4.33 \mathrm{~kg}$ / $\mathrm{m}^{2}$ and mean BSA was $1.99 \pm 0.17 \mathrm{~m}^{2}$. Mean A-a gradient was $3.68 \pm 1.77 \mathrm{kPa}$ and mean Horowitz quotient was $362.52 \pm 62.58 \mathrm{mmHg}$. There were 24 nonsmokers and nine smokers. five patients were ASA 1 status, 15 were ASA 2, 12 were ASA 3 and one patient was ASA 4. There was no statistically significant difference between groups in these parameters (Table 1).

No statistically significant difference was found between groups in baseline values of EIT measured parameters: nondependent/dependent $\mathrm{TV}$ ratio, horizontal $(\mathrm{hCoV})$ and vertical (vCoV) center of ventilation, GI, nondependent and dependent end-expiratory (EELI) and end-tidal (ETI) lung impedances (Table 1). There was no significant difference in baseline heart rate, mean arterial pressure and cardiac index, but patients in group PEEP 5 mbar had higher baseline stroke volume index (Table 1).

There was a statistically significant change of nondependent/dependent $\mathrm{TV}$ ratio between measurements, both in the whole cohort and between groups-patients who were ventilated with 0 or 5 mbar of PEEP had a significant shift of ventilation towards the nondependent lung after being positioned on their side ( $\mathrm{T} 1$ and $\mathrm{T} 2$ ) compared to patients who were ventilated with 10 mbar of PEEP (Table 2, Fig. 1). 
Table 1 Baseline patient characteristics

\begin{tabular}{|c|c|c|c|c|}
\hline & \multicolumn{3}{|l|}{ PEEP (mbar) } & \multirow[t]{2}{*}{$\mathrm{P}$} \\
\hline & 0 & 5 & 10 & \\
\hline Age & $60.5 \pm 11.7$ & $68.8 \pm 10.2$ & $57.0 \pm 14.6$ & 0.08 \\
\hline BMI $\left(\mathrm{kg} / \mathrm{m}^{2}\right)$ & $27.4 \pm 6.1$ & $28.2 \pm 2.6$ & $27.7 \pm 4.1$ & 0.29 \\
\hline $\operatorname{BSA}\left(\mathrm{m}^{2}\right)$ & $2.01 \pm 0.2$ & $1.9 \pm 0.1$ & $2.0 \pm 0.1$ & 0.31 \\
\hline A-a gradient $(\mathrm{kPa})$ & $3.6 \pm 2.1$ & $4.2 \pm 1.9$ & $3.2 \pm 1.2$ & 0.31 \\
\hline $\mathrm{PaO}_{2} / \mathrm{FiO}_{2}(\mathrm{mmHg})$ & $374.1 \pm 78.4$ & $338.2 \pm 56.8$ & $375.2 \pm 46.7$ & 0.25 \\
\hline Gender-male $(\mathrm{N}=23)$ & $8(34.8 \%)$ & $7(30.4 \%)$ & $8(34.8 \%)$ & 0.87 \\
\hline Gender-female $(\mathrm{N}=10)$ & $3(30 \%)$ & $4(40 \%)$ & $3(30 \%)$ & \\
\hline ASA I $(N=5)$ & $2(40 \%)$ & 0 & $3(60 \%)$ & 0.13 \\
\hline ASA II $(\mathrm{N}=15)$ & $3(20 \%)$ & $5(33.3 \%)$ & $7(46.7 \%)$ & \\
\hline ASA III (N=12) & $6(50 \%)$ & $5(41.7 \%)$ & $1(8.3 \%)$ & \\
\hline ASA IV $(\mathrm{N}=1)$ & 0 & $1(100 \%)$ & 0 & \\
\hline Smokers $(\mathrm{N}=9)$ & $4(44.4 \%)$ & $2(22.2 \%)$ & $3(33.3 \%)$ & 0.63 \\
\hline Non-smokers $(\mathrm{N}=24)$ & $7(29.2 \%)$ & $9(37.5 \%)$ & $8(33.3 \%)$ & \\
\hline Heart rate (bpm) & $75.3 \pm 15$ & $72.6 \pm 11.9$ & $70.4 \pm 9.8$ & 0.66 \\
\hline Mean arterial pressure $(\mathrm{mmHg})$ & $102.5 \pm 17.1$ & $103.1 \pm 14.3$ & $93.3 \pm 9.3$ & 0.12 \\
\hline Cardiac index $\left(1 / \mathrm{min} / \mathrm{m}^{2}\right)$ & $3.2 \pm 0.9$ & $4 \pm 1.5$ & $2.9 \pm 0.3$ & 0.09 \\
\hline Stroke volume index $\left(\mathrm{ml} / \mathrm{beat} / \mathrm{m}^{2}\right)$ & $42.5 \pm 8.6$ & $57.7 \pm 17.4$ & $42.9 \pm 8.1$ & $\mathbf{0 . 0 3}$ \\
\hline Non-dependent/dependent TV & $0.8 \pm 0.5$ & $0.8 \pm 0.5$ & $1.2 \pm 0.6$ & 0.11 \\
\hline Horizontal CoV (\%) & $48.9 \pm 13.8$ & $48.1 \pm 17.9$ & $49.4 \pm 7.9$ & 0.64 \\
\hline Vertical CoV (\%) & $52.2 \pm 6.1$ & $50.4 \pm 6.2$ & $62.6 \pm 38.6$ & 0.46 \\
\hline GI $(\%)$ & $58 \pm 20.6$ & $63.2 \pm 23.7$ & $61.9 \pm 26.8$ & 0.73 \\
\hline Nondependent EELI (AU) & $362 \pm 199$ & $344 \pm 233$ & $510 \pm 421$ & 0.60 \\
\hline Dependent EELI (AU) & $402 \pm 297$ & $459 \pm 305$ & $369 \pm 206$ & 0.68 \\
\hline Nondependent ETI (AU) & $1437 \pm 736$ & $1173 \pm 424$ & $1773 \pm 984$ & 0.32 \\
\hline Dependent ETI (AU) & $1822 \pm 944$ & $1664 \pm 643$ & $1727 \pm 832$ & 0.91 \\
\hline
\end{tabular}

Bold values indicate the statistically significant differences between groups

$B M I$ Body mass index, $B S A$ Body surface area, $T V$ Tidal variation, $C o V$ Center of ventilation, GI Global inhomogeneity index, EELI End-expiratory lung impedance, ETI End tidal lung impedance, $A U$ Arbitrary units

There was also a significant increase of A-a gradient in all patients, but it was even more pronounced in those ventilated with lower levels of PEEP (Table 2, Fig. 2). $\mathrm{PaO}_{2} / \mathrm{FiO}_{2}$ ratio had a significant change over time, but after post hoc correction was performed, no statistically significant difference was found between groups (Table 2, Fig. 3).

There was no statistically significant difference in changes of dynamic compliance between groups at T1 and T2 (Table 2, Fig. 4).

While there were significant differences found between hemodynamic measurements at various time points, we found no significant between-group differences in heart rate, MAP and CI, as well as in EIT derived parameters: hCOV, vCOV and GI index. We found significant difference in SVI between groups (Table 3 ).

A moderate, but statistically significant correlation between nondependent/dependent TV and A-a gradient both $5 \mathrm{~min}(\rho=0.52, \mathrm{p}<0.01)$ and $90 \mathrm{~min}$ after lateral positioning of the patient $(\rho=0.50, p<0.01)$ was found. GI index also shows a statistically significant correlation to A-a gradient which is even more pronounced $90 \mathrm{~min}$ after positioning $(\rho=0.84, \mathrm{p}<0.001)$ than $5 \mathrm{~min}(\rho=0.59, \mathrm{p}<0.01)$ after lateral positioning. Dynamic compliance showed no significant correlation to A-a gradient $(\rho=-0.20, \mathrm{p}=0.25$ in $\mathrm{T} 1$, $\rho=-0.32, p=0.07$ in $\mathrm{T} 2$ ).

\section{Discussion}

Results of this study showed that in patients that are mechanically ventilated in lateral decubitus position $10 \mathrm{mbar}$ of PEEP will lead to more symmetrical distribution of ventilation between left and right lung and improved alveolar gas exchange compared to 0 and 5 mbar of PEEP, without compromising hemodynamic stability. Dynamic compliance of the respiratory system does not correlate with alveoloarterial gradient in these patients, unlike EIT measured 
Table 2 Changes of nondependent/dependent TV, alveolo arterial (A-a) gradient, $\mathrm{PaO} 2 / \mathrm{FiO} 2$ ratio and dynamic compliance over time- $-\mathrm{T} 0$ : before induction of anaesthesia; T1: 5 min after lateral positioning; T2: 90 min after lateral positioning

\begin{tabular}{|c|c|c|c|c|c|}
\hline & \multicolumn{3}{|l|}{ PEEP } & \multirow[t]{2}{*}{$\mathrm{P}_{\mathrm{w}}$} & \multirow[t]{2}{*}{$\mathrm{P}_{\mathrm{b}}$} \\
\hline & 0 & 5 & 10 & & \\
\hline \multicolumn{6}{|c|}{ Nondependent/dependent TV } \\
\hline $\mathrm{T} 0$ & $0.79(0.12-1.47)$ & $0.79(0.14-1.44)$ & $1.2(0.55-1.85)$ & \multirow[t]{3}{*}{$<0.01$} & \multirow[t]{3}{*}{$\mathbf{0 . 0 3}$} \\
\hline $\mathrm{T} 1$ & $2.3(1.62-2.97)$ & $2.23(1.58-2.89)$ & $0.77(0.12-1.43)$ & & \\
\hline $\mathrm{T} 2$ & $3.3(2.61-3.97)$ & $2.52(1.86-3.17)$ & $0.91(0.26-1.57)$ & & \\
\hline \multicolumn{6}{|c|}{ A-a gradient $(\mathrm{kPa})$} \\
\hline T0 & $3.6(0.4-6.8)$ & $4.2(1.0-7.5)$ & $3.2(0-6.4)$ & \multirow[t]{3}{*}{0.02} & \multirow[t]{3}{*}{$\mathbf{0 . 0}$} \\
\hline $\mathrm{T} 1$ & $13.1(9.9-16.3)$ & $12.8(9.6-15.9)$ & $4.6(1.4-7.8)$ & & \\
\hline $\mathrm{T} 2$ & $15.1(11.9-18.3)$ & $16.4(13.2-19.6)$ & $10.2(7-13.4)$ & & \\
\hline \multicolumn{6}{|c|}{$\mathrm{PaO}_{2} / \mathrm{FiO}_{2}(\mathrm{mmHg})$} \\
\hline T0 & $374(322-425)$ & $338(286-389)$ & $375(323-426)$ & \multirow[t]{3}{*}{$<0.01$} & \multirow[t]{3}{*}{0.09} \\
\hline $\mathrm{T} 1$ & $386(334-437)$ & $389(337-440)$ & $515(463-566)$ & & \\
\hline $\mathrm{T} 2$ & $343(291-394)$ & $292(240-343)$ & $411(360-462)$ & & \\
\hline \multicolumn{6}{|c|}{ Dynamic compliance (ml/mbar) } \\
\hline $\mathrm{T} 1$ & $41(36-47)$ & $39(34-45)$ & $46(41-51)$ & \multirow[t]{2}{*}{0.12} & \multirow[t]{2}{*}{0.49} \\
\hline $\mathrm{T} 2$ & $39(34-45)$ & $42(37-48)$ & $45(40-50)$ & & \\
\hline
\end{tabular}

Bold values indicate the statistically significant differences between groups

Values are expressed as mean and 95\% confidence interval

Two-factor repeated measures ANOVA. $\mathrm{P}_{\mathrm{w}}$-within group interactions, $\mathrm{P}_{\mathrm{b}}$ - between groups interactions with post hoc Bonferroni correction

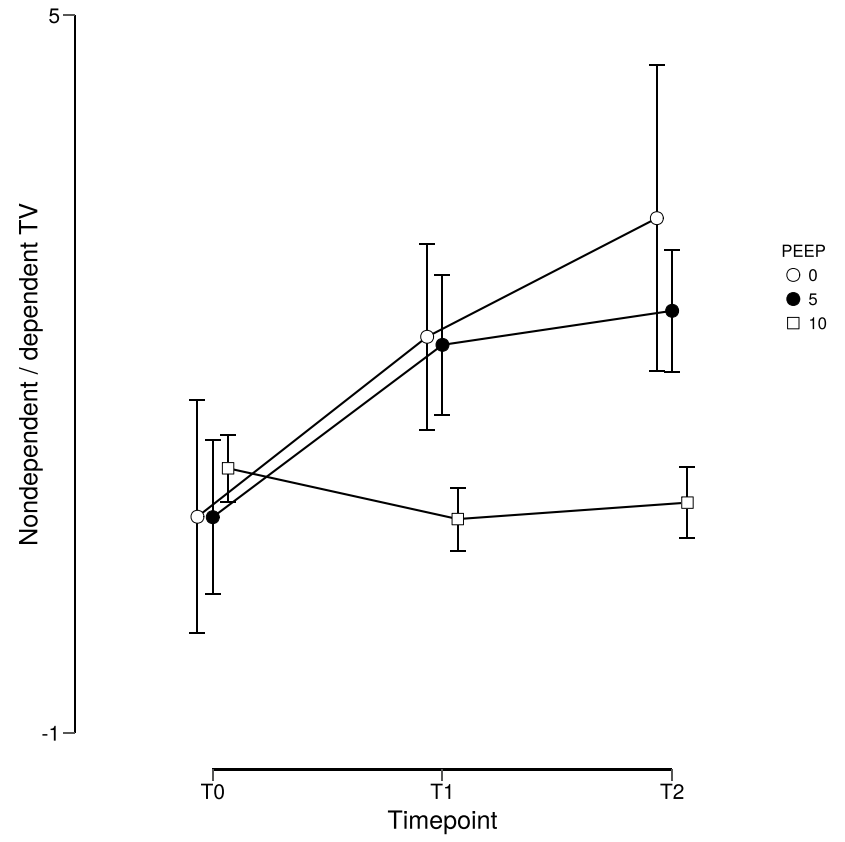

Fig. 1 Changes of nondependent/dependent TV over time; T1: $5 \mathrm{~min}$ after lateral positioning; T2: 90 min after lateral positioning

parameters: non-dependent/dependent TV ratio and GI index which showed a moderate but significant correlation.

Lateral patient positioning during general anesthesia leads to drop of thoracic compliance [24], which in certain scenarios might cause barotrauma in volume controlled or hypoventilation in pressure controlled ventilation, depending

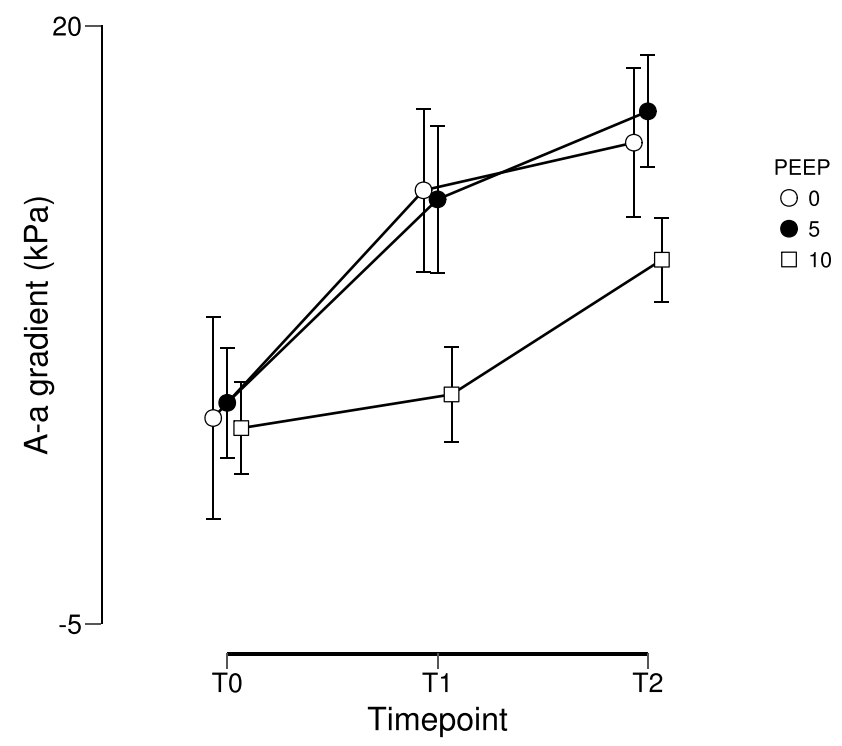

Fig. 2 Changes of alveolo arterial (A-a) gradient over time; T1: 5 min after lateral positioning; T2: 90 min after lateral positioning

on ventilator settings and alarm limits. In supine patients, pressure of abdominal organs on the diaphragm and thorax is generally equally distributed between left and right lung, and while there are conflicting opinions of using respiratory system compliance as a guiding parameter in setting the "optimal" PEEP level [25], Cordeiro et al. have shown that there is a strong correlation between respiratory system compliance and oxygenation levels $(\mathrm{r}=0.8, \mathrm{p}<0.001)$ in mechanically ventilated cardiac surgical patients (which 


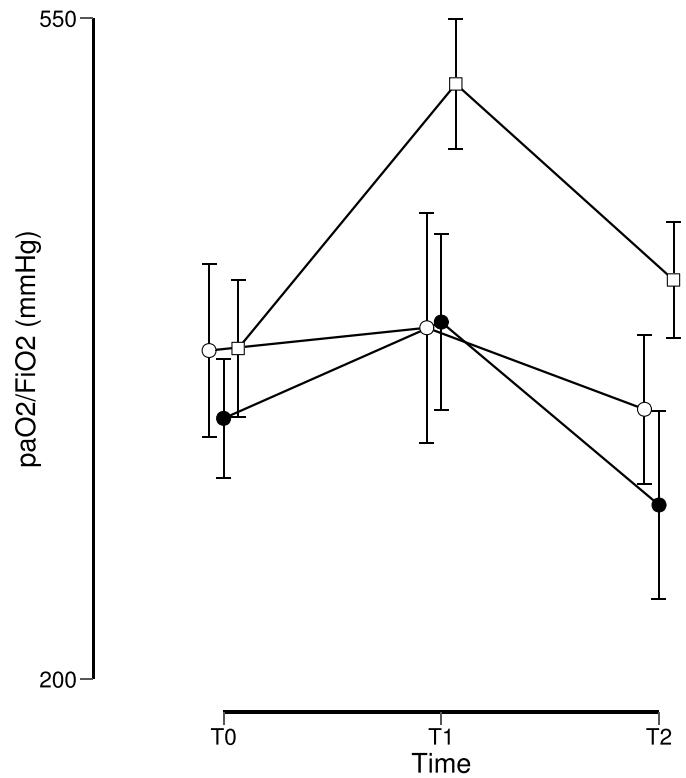

Fig. 3 Changes of $\mathrm{PaO}_{2} / \mathrm{FiO}_{2}$ ratio over time; T1: 5 min after lateral positioning; T2: $90 \mathrm{~min}$ after lateral positioning

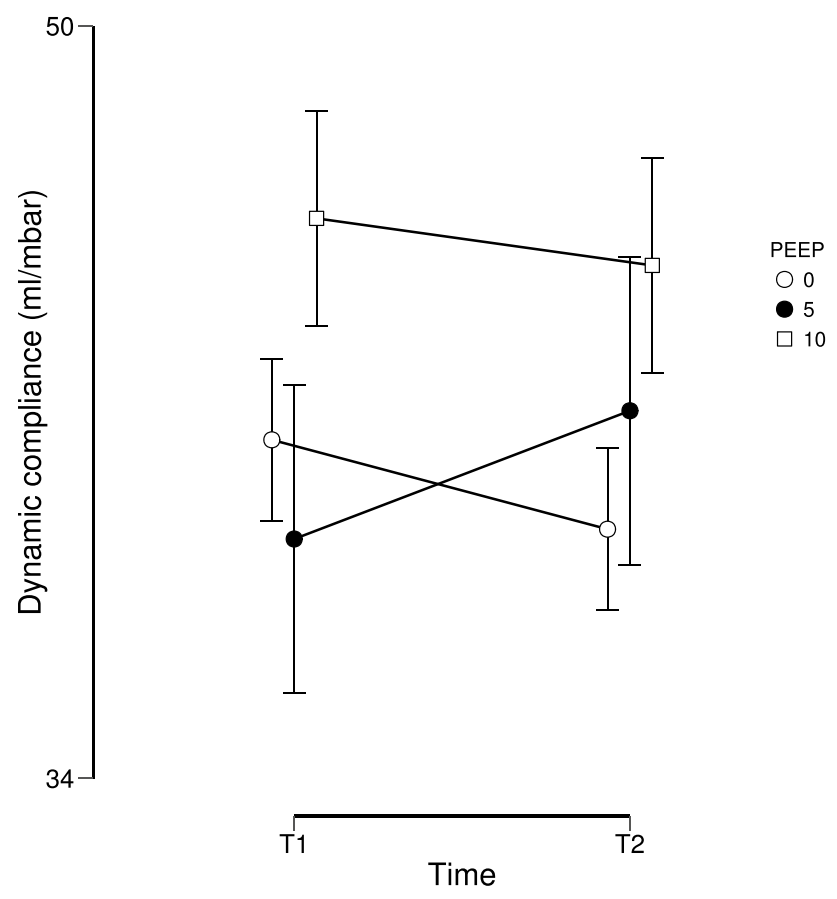

Fig. 4 Changes in dynamic compliance over time; T1: 5 min after lateral positioning; T2: $90 \mathrm{~min}$ after lateral positioning

are positioned in supine position after surgery) [26]. However, in patients that are ventilated in lateral position (i.e. patients that were subjects in this study), there is a significant gradient in regional compliance which is present in both ventral-dorsal and left-right axis. Because of that, global compliance does not accurately represent all lung regions,
Table 3 Changes of heart rate (HR), mean arterial pressure (MAP), cardiac index (CI), stroke volume index (SVI), horizontal (hCov) and vertical $(\mathrm{vCoV})$ center of ventilation and GI over time-T0: before induction of anaesthesia; T1: $5 \mathrm{~min}$ after lateral positioning; T2: 90 min after lateral positioning

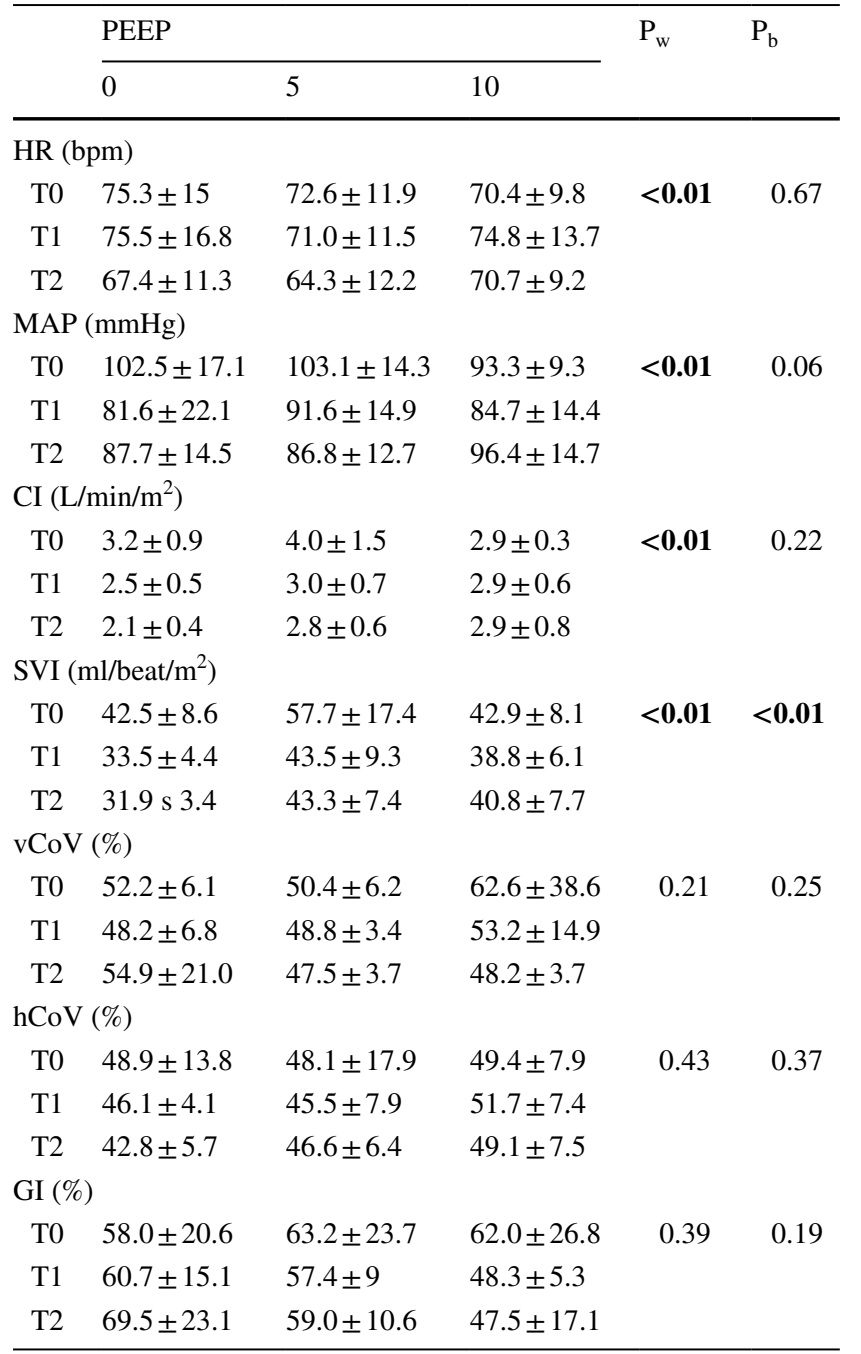

Bold values indicate the statistically significant differences between groups

Values are expressed as mean and standard deviation

Two-factor repeated measures ANOVA. $\mathrm{P}_{\mathrm{w}}$-within group interactions, $\mathrm{P}_{\mathrm{b}}$ - between groups interactions with post hoc Bonferroni correction

and results of this study show that compliance doesn't correlate with regional distribution of ventilation and parameters of alveolar gas exchange. With EIT, compliance can be measured for each pixel (and subsequently for lung regions) by dividing tidal impedance variation with driving pressure, as was demonstrated by Costa et al. [27].

According to the results obtained in this study, patients ventilated with lower levels of PEEP ( 0 and 5 mbar) will develop shunting caused by alveolar collapse of the dependent lung (as shown by increased nondependent/dependent 
TV ratio), which is demonstrated by worsening of A-a gradient that is more profound in these two groups compared to the 10 mbar group.

Experimental model of induced ARDS in rats which were ventilated in lateral position and two PEEP levels ( 3 and 10 mbar) has shown that test subjects with lower PEEP levels will develop histological signs of alveolar instability after 75 min of mechanical ventilation, but without differences in arterial $\mathrm{PO}_{2}$ between groups [28].

In a heterogenous population of ICU patients with various lung pathologies as well as patients with healthy lungs, lateral positioning has shown no effect on parameters of alveolar gas exchange, but has decreased lung compliance in patients with healthy lungs [29]. In patients with ARDS, lateral positioning increases both airway resistance and lung elastance [30].

Effect of higher PEEP levels on hemodynamics have been extensively studied, and general consensus is that PEEP may worsen hemodynamic instability, especially in critically ill patients [31-33]. However, results of this study show that PEEP of 10 mbar is perfectly safe in tested subjects. The reason for that might be that increase of PEEP will be more deleterious in patients that are volume (be it absolute or relative) depleted. Intraoperative volume maintenance and replacement in test subjects of this study was guided by pulse pressure variation, a dynamic parameter which is very sensitive and specific in discriminating volume responders and non-responders [34], and because of that patients that were included in study tolerated increased PEEP well.

Center of ventilation indices which were used in this study were calculated without image segmentation. Several different methods of center of ventilation calculation have been described [16], and patients that have pronounced ventilation asymmetry (such as patients with increased nondependent/dependent TV ratio) might have larger differences in $\mathrm{CoV}$ values compared to those calculated with our method. Although both the nondependent/dependent TV ratio and $\mathrm{hCoV}$ attempt to quantify distribution of ventilation along the latero-lateral axis, method of calculation is different. While $\mathrm{hCoV}$ is calculated from individual pixel impedance variation in each row and must be made offline after data export, nondependent/dependent TV ratio is calculated from TV percentages divided across ROI and is easily calculated by the bedside. Since the nondependent/dependent TV ratio is calculated by dividing nondependent and dependent TV percentages, differences in calculated value between patients are much greater compared to the $\mathrm{hCoV}$ value.

There were certain study shortcomings. Dynamic compliance was used instead of static compliance because it is technically challenging to perform inspiratory hold maneuver need to measure plateau pressure on the anesthesia machine. However, since patients with obstructive lung disease have been excluded from this study, their peak inspiratory pressure does not differ much from plateau pressure and therefore dynamic compliance which was calculated should not deviate too much from static compliance. The choice of hemodynamic monitoring method which was used due to financial constraints (pulse wave transit time derived cardiac output) is not validated as well as some other minimally invasive measurement methods (such as arterial waveform analysis systems) which have been extensively tested [35], however since changes in hemodynamic parameters were measured, and not absolute values, this potential shortcoming should not present a problem in result interpretation.

\section{Conclusion}

Our findings suggest that, when compared to PEEP levels of 0 or $5 \mathrm{mbar}$, a PEEP level of 10 mbar leads to improved gas exchange in the lungs and more symmetrical ventilation distribution between left and right lung without compromising hemodynamic stability. Due to inhomogeneous lung mechanics and poor correlation of respiratory system compliance with parameters of alveolar gas exchange EIT could be a useful method for PEEP titration in these patients. Further studies are needed to assess changes of distribution of ventilation in patients with lung pathology who are ventilated in lateral position.

Acknowledgements The authors would like to thank Dr. Tobias Becher for providing the .xls (Microsoft Excel) files used to calculate center of ventilation and GI index.

\section{Compliance with ethical standards}

Conflict of interest The authors declare that they have no conflict of interest.

Ethical approval All procedures performed in studies involving human participants were in accordance with the ethical standards of the institutional and/or national research committee and with the 1964 Helsinki declaration and its later amendments or comparable ethical standards.

Informed consent Informed consent was obtained from all individual participants included in the study.

\section{References}

1. Butterworth JF, Mackey DC, Wasnick JD. Anesthesia for thoracic surgery. In: Morgan \& Mikhail's clinical anesthesiology. New York: Mcgraw-Hill; 2013. p. 545-573.

2. Baumgardner JE, Hedenstierna G. Ventilation/perfusion distributions revisited. Curr Opin Anaesthesiol. 2016;29:2-7.

3. Zavorsky GS, Hoffman SL. Pulmonary gas exchange in the morbidly obese. Obes Rev. 2008;9:326-39.

4. Gattinoni L, Carlesso E, Cressoni M. Selecting the 'right' positive end-expiratory pressure level. Curr Opin Crit Care. 2015;21:50-7. 
5. Blankman P, Hasan D, Groot Jebbink E, et al. Detection of 'best' positive end-expiratory pressure derived from electrical impedance tomography parameters during a decremental positive endexpiratory pressure trial. Crit Care. 2014;18:R95.

6. Suter PM, Fairley B, Isenberg MD. Optimum end-expiratory airway pressure in patients with acute pulmonary failure. $\mathrm{N}$ Engl $\mathrm{J}$ Med. 1975;292:284-9.

7. ARDSNet. Ventilation with lower tidal volumes as compared with traditional tidal volumes for acute lung injury and the acute respiratory distress syndrome. N Engl J Med. 2000;342:1301-8.

8. Bouhemad B, Brisson H, Le-Guen M, et al. Bedside ultrasound assessment of positive end-expiratory pressure-induced lung recruitment. Am J Respir Crit Care Med. 2011;183:341-7.

9. Liu S, Tan L, Möller K, et al. Identification of regional overdistension, recruitment and cyclic alveolar collapse with electrical impedance tomography in an experimental ARDS model. Crit Care. 2016;20(1):119.

10. Zhao Z, Pulletz S, Frerichs I, et al. The EIT-based global inhomogeneity index is highly correlated with regional lung opening in patients with acute respiratory distress syndrome. BMC Res Notes 7. 2014;1:82. https://doi.org/10.1186/1756-0500-7-82.

11. Spadaro S, Mauri T, Böhm SH, et al. Variation of poorly ventilated lung units (silent spaces) measured by electrical impedance tomography to dynamically assess recruitment. Crit Care. 2018;22(1):26. https://doi.org/10.1186/s13054-017-1931-7.

12. He X, Jiang J, Liu Y, et al. Electrical impedance tomographyguided PEEP titration in patients undergoing laparoscopic abdominal surgery. Medicine (Baltimore). 2016;95:e3306.

13. Karsten J, Luepschen H, Grossherr M, et al. Effect of PEEP on regional ventilation during laparoscopic surgery monitored by electrical impedance tomography. Acta Anaesthesiol Scand. 2011;55:878-86.

14. Meier T, Leibecke T, Eckmann C, et al. Electrical impedance tomography: changes in distribution of pulmonary ventilation during laparoscopic surgery in a porcine model. Langenbeck's Arch Surg. 2006;391:383-9.

15. Reinius H, Borges JB, Fredén F, et al. Real-time ventilation and perfusion distributions by electrical impedance tomography during one-lung ventilation with capnothorax. Acta Anaesthesiol Scand. 2015;59:354-68.

16. Sobota V, Roubik K. Center of ventilation-methods of calculation using electrical impedance tomography and the influence of image segmentation. IFMBE Proc. 2016;57:1258-63.

17. Becher T, Kott M, Schädler D, et al. Influence of tidal volume on ventilation inhomogeneity assessed by electrical impedance tomography during controlled mechanical ventilation. Physiol Measurement. 2015;36(6):1137. https://doi. org/10.1088/0967-3334/36/6/1137.

18. Frerichs I, Dargaville PA, van Genderingen H, et al. Lung volume recruitment after surfactant administration modifies spatial distribution of ventilation. Am J Respir Crit Care Med. 2006;174:772-9.

19. Yun L, He HW, Möller K, et al. Assessment of lung recruitment by electrical impedance tomography and oxygenation in ARDS patients. Medicine (Baltimore). 2016;95:1-9.
20. Faul F, Erdfelder E, Buchner A, et al. G*Power 3: a flexible statistical power analysis program for the social, behavioral and biomedical sciences. Behav Res Methods. 2009;41:1149-60.

21. JASP, Team. JASP v 0.9.1. http://www.jasp-stats.org (2018).

22. Jamovi, Team. jamovi v0.9.5. http://www.jamovi.org (2018).

23. Hammer O, Harper DAT, Ryan PD. PAST: paleontological statistics software package for education and data analysis. Paleontol Electron. 2001;4:9.

24. Szkulmowski Z, Robert D. Change of the respiratory system compliance between supine and lateral position. Now Lek. 2005;74:317-24.

25. Kallet RH. Should PEEP titration be based on chest mechanics in patients with ARDS? Respir Care. 2016;61:876-90.

26. Cordeiro ALL, de Oliveira LF, Queiroz TC, et al. Association of respiratory mechanics with oxygenation and duration of mechanical ventilation after cardiac surgery. Int J Cardiovasc Sci. 2018;31:244-9.

27. Costa ELV, Borges JB, Melo A, et al. Bedside estimation of recruitable alveolar collapse and hyperdistension by electrical impedance tomography. Intensive Care Med. 2009;35:1132-7.

28. Pavone L, Albert S, DiRocco J, et al. Alveolar instability caused by mechanical ventilation initially damages the nondependent normal lung. Crit Care. 2007;11:R104.

29. Thomas PJ, Paratz JD, Lipman J, et al. Lateral positioning of ventilated intensive care patients: a study of oxygenation, respiratory mechanics, hemodynamics, and adverse events. Hear Lung. 2007;36:277-86.

30. Riad Z, Mezidi M, Subtil F, et al. Short-term effects of the prone positioning maneuver on lung and chest wall mechanics in patients with acute respiratory distress syndrome. Am J Respir Crit Care Med. 2018;197:1355-8.

31. Michard F, Chemla D, Richard C, et al. Clinical use of respiratory changes in arterial pulse pressure to monitor the hemodynamic effects of PEEP. Am J Respir Crit Care Med. 1999;159:935-9.

32. Luecke T, Pelosi P. Clinical review: positive end-expiratory pressure and cardiac output. Crit Care. 2005;9:607-21.

33. Vieillard-Baron A, Matthay M, Teboul JL, et al. Experts' opinion on management of hemodynamics in ARDS patients: focus on the effects of mechanical ventilation. Intensive Care Med. 2016;42(5):739. https://doi.org/10.1007/s00134-016-4326-3.

34. Cannesson M, Musard H, Desebbe O, et al. The ability of stroke volume variations obtained with vigileo/flotrac system to monitor fluid responsiveness in mechanically ventilated patients. Anesth Analg. 2009;108:513-7.

35. Slagt C, Malagon I, Groeneveld ABJ. Systematic review of uncalibrated arterial pressure waveform analysis to determine cardiac output and stroke volume variation. Br J Anaesth. 2014;112:626-37.

Publisher's Note Springer Nature remains neutral with regard to jurisdictional claims in published maps and institutional affiliations. 